\title{
Maximum Velocity for Matter in Relation to the Schwarzschild Radius Predicts Zero Time Dilation for Quasars
}

\author{
Espen Gaarder Haug* \\ Norwegian University of Life Sciences
}

October 28,2018

\begin{abstract}
This is a short note on a new way to describe Haug's newly introduced maximum velocity for matter in relation to the Schwarzschild radius. This leads to a probabilistic Schwarzschild radius for elementary particles with mass smaller than the Planck mass. In addition, our maximum velocity, when linked to the Schwarzschild radius, seems to predict that particles just at that radius cannot move. This implies that radiation from the Schwarzschild radius not can undergo velocity time dilation. Our maximum velocity of matter, therefore, seems to predict no time dilation, even in high $\mathrm{Z}$ quasars, as has surprisingly been observed recently.
\end{abstract}

Keywords: Schwarzschild radius, maximum velocity of matter, probabilistic Schwarzschild radius, quasars, time dilation.

\section{Haug's Maximum Velocity for Matter}

Haug's newly introduced maximum velocity for matter has been published in a series of papers and working papers; see, for example $[1,2,3]$. It is given by

$$
v_{\max }=c \sqrt{1-\frac{l_{p}^{2}}{\bar{\lambda}^{2}}}
$$

where $l_{p}$ is the Planck length; see also $[4,5]$. This formula can be derived simply by putting a Planck length limit on length contraction, or one can set the maximum relativistic mass (energy) for an elementary particle to the Planck mass (energy), for example. Alternatively, one can even derive this formula from Heisenberg's uncertainty principle, if one assumes that the minimum uncertainty in position is the Planck length, see [6].

Haug has also recently shown that the Planck length is given by

$$
l_{p}=\sqrt{\frac{1}{2} R_{s} \bar{\lambda}}
$$

where $R_{s}$ is the Schwarzschild radius and $\bar{\lambda}$ is the reduced Compton wavelength of the same mass. An important point is that the Schwarzschild radius and the reduced Compton wavelength can be found independent of any knowledge of Newton's gravitational constant or any knowledge of the Planck constant as shown by [7]. This means the maximum velocity is given by

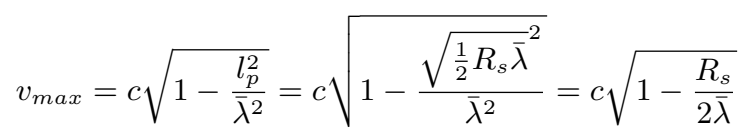

We think this formula only gives meaning for elementary particles; we have suggested in other working papers that all elementary particles have a probabilistic Schwarzschild radius. More precisely, they have a Schwarzschild radius equal to the Planck length with a frequency (probability) of only $\frac{l_{p}}{\lambda}$. That is to say, the probabilistic Schwarzschild radius for an elementary particle is

$$
R_{s}=2 l_{p} \frac{l_{p}}{\bar{\lambda}}
$$

*e-mail espenhaug@mac.com. Thanks to Victoria Terces for helping me edit this manuscript. 
This version of the Schwarzschild radius formula also holds for masses larger than the Planck mass, but then the interpretation is no longer simply probabilistic, as the probabilistic factor (frequency): $\frac{l_{p}}{\lambda}$ will be higher than one. Integer numbers above one indicate the number of Planck masses, and there is certainty for Planck masses. The fraction above one indicates an additional probabilistic factor for the remaining mass. This means that probabilistic effects dominate below the Planck mass and determinism dominates above; see [6].

Be aware that the Schwarzschild radius of any mass, including the probabilistic Schwarzschild radius of elementary particles such as electrons, can be found independent of any knowledge of $G$. The Planck length can also be found independent of $G$ and $\hbar$; see Haug's recent working papers [8].

Even if the maximum velocity formula only holds for elementary particles, it can give a good indication of the maximum velocity of composite masses (e.g. particles such as protons). As suggested by [2,6], the maximum velocity of the composite object will be limited by the heaviest elementary particle in the object. Our theory predicts that the heaviest particle will start to dissolve into energy when it reaches the maximum velocity. Any elementary particle is then a Planck mass

\section{Gravitational Time Dilation Plus Velocity Time Dilation at the Schwarzschild Radius For a Planck Mass Gravitational Object}

If we take into account gravitational time dilation plus velocity time dilation from general relativity utilizing the Schwarzschild metric, we have (see [9] for an excellent introduction to this ${ }^{1}$ )

$$
d \tau=\sqrt{1-\frac{R_{s}}{r}-\frac{v^{2}}{c^{2}}}
$$

where $R_{s}$ is the Schwarzschild radius, and $r=R+h$ where $h$ is the height (distance) from $R$. Further, $v$ is the orbital velocity of the object traveling around the gravitational object. Assume we now want to look at the special case of a Planck mass particle. This is the smallest known mass with a Schwarzschild radius. A particle with a smaller mass will have a Schwarzschild radius less than the Planck length according to the standard formula of

$$
R_{s}=\frac{2 G m}{c^{2}}
$$

that again is equivalent to $R_{s}=2 l_{p} \frac{l_{p}}{\lambda}$. We could get into a lengthy discussion over whether the 2 factor actually should be 1 instead of 2 in a very strong gravitational field, but that is beyond the scope of this short paper. Instead, let us assume a gravitational object with Planck mass $m_{p}$ and that something is moving just at the Schwarzschild radius. In other words, we assume $h=0$ and $r=R_{s}+0$ this leads to

$$
d \tau=\sqrt{1-\frac{R_{s}}{R_{s}}-\frac{v^{2}}{c^{2}}}
$$

As $\frac{R_{s}}{R_{s}}=1$, this leads to the square root of a negative number for any $v>0$. Does this mean that the time dilation at the Schwarzschild radius is imaginary? We don't think so. However, the maximum velocity, as given by Haug's maximum velocity formula for a Planck mass particle, is

$$
v_{\max }=c \sqrt{1-\frac{l_{p}^{2}}{\bar{\lambda}^{2}}}=\sqrt{1-\frac{l_{p}^{2}}{\bar{\lambda}^{2}}}=\sqrt{1-\frac{l_{p}^{2}}{l_{p}^{2}}}=0
$$

where $\bar{\lambda}$ is the reduced Compton wavelength of the particle in question. For any observed elementary particle, such as an electron, this velocity is very close to $c$, but far above what has been accomplished by the Large Hadron Collider. In the special case of a Planck mass particle, we have $\bar{\lambda}=l_{p}$; this means the maximum velocity for a Planck mass particle is zero. Thus, for a Planck mass particle we must have

\footnotetext{
${ }^{1}$ p. 175
} 


$$
\begin{aligned}
& d \tau=\sqrt{1-\frac{R_{s}}{R_{s}}-\frac{v_{\text {max }}^{2}}{c^{2}}} \\
& d \tau=\sqrt{1-\frac{R_{s}}{R_{s}}-\frac{\left(c \sqrt{1-\frac{l_{p}^{2}}{l_{p}^{2}}}\right)^{2}}{c^{2}}} \\
& d \tau=\sqrt{1-1-\frac{c^{2}\left(1-\frac{l_{p}^{2}}{l_{p}^{2}}\right)}{c^{2}}} \\
& d \tau=\sqrt{-1+\frac{l_{p}^{2}}{l_{p}^{2}}}=0
\end{aligned}
$$

That is to say, we have zero time dilation at the Schwarzschild radius for a Planck mass. It is not a new concept that time must stand still at the Schwarzschild radius. However, what we have not seen discussed before is that any object (with mass) at the Schwarzschild radius must itself stand still in order for this to happen. This is consistent with Haug's predictions where he has claimed that the Planck mass particle must stand absolutely still, see $[2,6]$. However, it only stands still for one Planck second before it bursts into energy. We can think of standard elementary particles moving at very close to the speed of light towards the Schwarzschild radius. They are getting dramatically compressed, due to the very strong gravitational field. Just at the Schwarzschild radius, they are compressed to Planck mass particles

$$
\frac{m}{\sqrt{1-\frac{v_{\max }^{2}}{c^{2}}}}=\frac{\frac{\hbar}{\bar{\lambda} \frac{1}{c}}}{\sqrt{1-\frac{\left(c \sqrt{1-\frac{l_{p}^{2}}{\bar{\lambda}^{2}}}\right)^{2}}{c^{2}}}}=\frac{\hbar}{l_{p}} \frac{1}{c}=m_{p}
$$

The Planck mass particle then suddenly stand still for one Planck second before they burst into energy. One could imagine some of this energy leaving the Schwarzschild radius in sudden bursts of energy. This leads to a breakdown of Lorentz symmetry at the Planck scale. A breakdown in Lorentz symmetry is not that controversial, as it is also predicted by several quantum gravity theories; see [10, 11, 12]. Alternatively, one needs to introduce imaginary time dilation (the square root of negative one), but this does not seem to be very logical.

In modern cosmology, it is commonly assumed that a quasar is supermassive object, often called a "black hole." Further, it is assumed that the radiation from the quasar likely comes from a layer of matter close to the black hole. If quasars are radiating black holes, then even high $\mathrm{Z}$ quasars will likely not have any time dilation. This because it is just at the Schwarzschild radius that the particles burst into energy after standing still for one Planck second.

This prediction of no velocity time dilation in quasars (black holes) is exactly what modern observational physics has shown [13, 14]. Hawking studied a series of high red-shift (high Z) quasars and surprisingly did not find the expected time dilation predicted by standard physics. observational study is a great achievement, but we are still critical towards postulates associated with the growth of the central supermassive black hole in quasars/active galactic nucleus (AGN) to offset the missing expected time dilation in the standard theory. Could this also mean there is a very different explanation for cosmological red-shift than today's theory claims? At a minimum, it suggests that we should, once again, study other alternative theories in more detail. One could even suggest that the lack of time dilation in quasars is one of the first observations possibly is confirming that Lorentz symmetry breaks at the Planck scale, the Schwarzschild radius is the Planck scale.

Our newly introduced maximum velocity also indicates so called black holes are possibly a misnomer. All elementary particles must be compressed to Planck mass particles at Schwarzschild radius as they then reaches the velocity of $v_{\max }=c \sqrt{1-\frac{R_{s}}{2 \lambda}}=c \sqrt{1-\frac{l_{p}^{2}}{\lambda^{2}}}$, they then stand still and then burst into energy. So they go from a velocity just below the speed of light to standing still for one Planck second and then bursting into energy and moving at speed $c$. Some of this energy likely goes inward into the so-called black hole, so in this sense it is black, but some of it likely also bursts out. Remember this is at the very boundary of the black hole. This indicates the Schwarzschild radius is a radiation horizon where matter is compressed and then bursts into energy. Black holes are, for this reason, likely white holes, and one will not observe any time dilation from the radiation coming from the Schwarzschild radius itself, and very little time dilation from particles at a distance of a few Planck lengths away from the the Schwarzschild radius. 


\section{Heisenberg Uncertainty Principle Extended to the Schwarzschild Radius}

In 1927, Heisenberg [15] published what today is known as the Heisenberg Schwarzschild principle; (see also [16])

$$
\Delta p \Delta x \geq \hbar
$$

In 1932, during his Nobel lecture Heisenberg himself suggested the existence of a universal least length. Later on (in 1958), he assumed that the least length was the atom nuclei diameter $10^{-15} \mathrm{~m}$; see also [17]. A series of researchers have argued that the Planck length is indeed the shortest length that gives any meaning; see [18, 19], for example. Modern quantum gravity theories are also returning to the idea that the Planck length is the universal least length, even though there is still considerable scientific debate on this.

Here we will assume that the smaller possible uncertainty in the position is the Planck length. We can then replace our expression for the Planck length with $l_{p}=\sqrt{\frac{1}{2} R_{s} \bar{\lambda}}$ and see what we get. Further, we will assume that elementary particles must have a momentum smaller than or equal to the Planck mass momentum; the maximum momentum is then $m_{p} c=\frac{\hbar}{l_{p}}$. If this is the maximum momentum of an elementary particle (including the Planck mass particle), then this must also be the maximum uncertainty in the momentum for an elementary particle. Based on this we get

$$
\begin{aligned}
\Delta p \Delta x & \geq \hbar \\
\Delta p l_{p} & \geq \hbar \\
\Delta p \sqrt{\frac{1}{2} R_{s} \bar{\lambda}} & \geq \hbar \\
\sqrt{\frac{1}{2} R_{s}} & \geq \frac{\hbar}{\Delta p \sqrt{\bar{\lambda}}} \\
\sqrt{\frac{1}{2} R_{s}} & \geq \frac{\hbar}{\Delta m_{p} c \sqrt{\bar{\lambda}}} \\
\sqrt{\frac{1}{2} R_{s}} & \geq \frac{\hbar}{\frac{\hbar}{l_{p}} \frac{1}{c} c \sqrt{\bar{\lambda}}} \\
\sqrt{\frac{1}{2} R_{s}} & \geq \frac{l_{p}}{\sqrt{\bar{\lambda}}} \\
R_{s} & \geq 2 \frac{l_{p}^{2}}{\bar{\lambda}}=2 l_{p} \frac{l_{p}}{\bar{\lambda}}
\end{aligned}
$$

The correct interpretation of this is likely that the Schwarzschild radius is probabilistic for elementary particles. All elementary particles have a Schwarzschild radius equal to the Planck length, but they only have this at the Compton periodicity, and the Schwarzschild radius only lasts for one Planck second for each unit of Compton time. That is the Schwarzschild radius is the reduced Compton frequency over the shortest possible time interval multiplied by the shortest possible length. This means the probability inside a Planck second for observing the Schwarzschild radius is $\frac{l_{p}}{\bar{\lambda}}$. For the Planck mass particle (also known by modern physics as a micro black hole), this probability is one, because we then have $\bar{\lambda}=l_{p}$. That is why the Planck mass particle is the smallest mass that has a Schwarzschild radius. Smaller particles still have a Schwarzschild radius, but it is then probabilistic.

\section{Conclusion}

In this paper, we have shown that there is a link between the Schwarzschild radius and our maximum velocity of matter. An important point is that the Schwarzschild radius can be found independent of any knowledge of Newton's gravitational constant or any knowledge of the Planck constant. This again leads to the idea that we can derive a probabilistic Schwarzschild radius for elementary particles using the Heisenberg uncertainty principle.

Our maximum velocity of matter, when linked to the Schwarzschild radius, also seems to predict that even high $\mathrm{Z}$ quasars cannot have time dilation, something that has been confirmed by experiments. 


\section{References}

[1] E. G. Haug. The gravitational constant and the Planck units. A simplification of the quantum realm. Physics Essays Vol 29, No 4, 2016.

[2] E. G. Haug. The ultimate limits of the relativistic rocket equation. The Planck photon rocket. Acta Astronautica, 136, 2017.

[3] E. G. Haug. Newton and Einstein's gravity in a new perspective for Planck masses and smaller sized objects. International Journal of Astronomy and Astrophysics, 2018.

[4] Max Planck. Naturlische Maasseinheiten. Der Königlich Preussischen Akademie Der Wissenschaften, p. 479., 1899.

[5] Max Planck. Vorlesungen über die Theorie der Wärmestrahlung. Leipzig: J.A. Barth, p. 163, see also the English translation "The Theory of Radiation" (1959) Dover, 1906.

[6] E. G. Haug. Finding the planck length independent of newton's gravitational constant and the Planck constant: The Compton clock model of matter. https://www.preprints.org/manuscript/201809.0396/v1, 2018.

[7] E. G. Haug. Revisiting the derivation of Heisenberg's uncertainty principle: The collapse of uncertainty at the Planck scale. preprints.org, 2018.

[8] E. G. Haug. Gravity without Newton's gravitational constant and no knowledge of mass size. preprints.org, 2018.

[9] Ø Grøn. Lecture Notes on the General Theory of Relativity. Springer Verlag, 2009.

[10] F. Kislat and H. Krawczynski. Planck-scale constraints on anisotropic Lorentz and CPT invariance violations from optical polarization measurements. Physical Review D, 95, 2017.

[11] G. Amelino-Cameliaa, B. J. Ellisc, N.E. Mavromatosa, D.V. Nanopoulosd, and Sarkar S. Potential sensitivity of gamma-ray burster observations to wave dispersion in vacuo. Nature, 393, 2998.

[12] Reyesa C. M., S. Ossandonb, and C. Reyesc. Higher-order Lorentz-invariance violation, quantum gravity and fine-tuning. Physics Letters B, 746, 2005.

[13] M. R. S. Hawkings. On time dilation in quasar light curves. Mon. Not. R. Astron. Soc., 406:10471054, 2000.

[14] M. R. S. Hawkings. Time dilation and quasar variability. The Astrophysical Journal, 553:97-100, 2001.

[15] W. Heisenberg. Über den anschaulichen inhalt der quantentheoretischen kinematik und mechanik. Zeitschrift für Physik, (43):172-198, 1927.

[16] E. H.. Kennard. Zur quantenmechanik einfacher bewegungstypen. Zeitschrift für Physik, (44):326$352,1927$.

[17] R. L. Wadlinger and Hunter G. Max Planck's natural units. The Physics Teacher, pages 528-529, 1988.

[18] T. Padmanabhan. Planck length as the lower bound to all physical length scales. General Relativity and Gravitation, 17, 1985.

[19] R. J. Adler. Six easy roads to the Planck scale. American Journal of Physics, 78(9), 2010. 